\title{
REATOR ELETRÔNICO AUTO-OSCILANTE COM CONTROLE DE INTENSIDADE LUMINOSA
}

\author{
Álysson R. Seidel ${ }^{*}$ \\ Humberto Pinheiro *
}

Fábio E. Bisogno *

Ricardo N. Prado*

rnprado@ieee.org

*GEDRE, PPGEE, UFSM, CEP 97105-900 - Santa Maria - RS, Brasil

\begin{abstract}
This paper presents a simple alternative of electronic ballast operating in self-sustained oscillating mode with dimming capability for fluorescent lamps. A simple modification in the gate driver circuit allows the lamp to dim without compromising the simplicity, reliability, and low cost which characterize the self-oscillating electronic ballast. A qualitative analysis is presented to explain the behavior of the proposed self-oscillating electronic ballast with dimming feature. In addition the stability and the key equations for the design are derived using the extended Nyquist criterion and describing function method. Experimental results are presented to demonstrate the performance and to validate the analysis carried out.
\end{abstract}

KEYWORDS: Self-oscillating, electronic ballast, fluorescent lamps, dimming.

\section{RESUMO}

Este artigo apresenta um reator eletrônico autooscilante com variação de intensidade luminosa de lâmpadas fluorescentes. Uma simples alteração no circuito de comando permite controlar a intensidade luminosa da lâmpada sem comprometer as características do reator

Artigo submetido em 26/02/02

1a. Revisão em 26/08/02

Aceito sob recomendação do Ed. Assoc. Prof. Antonio M. N. Lima que são sua simplicidade, confiabilidade e baixo custo. É feita uma análise qualitativa do reator eletrônico autooscilante com variação de intensidade luminosa. Além disso, faz-se a análise de estabilidade e são obtidas equações de projeto derivadas de métodos como função descritiva e o critério estendido de estabilidade de Nyquist. Resultados experimentais são apresentados no intuito de demonstrar e validar a análise realizada.

PALAVRAS-CHAVE: Auto-Oscilante, reator eletrônico, iluminação fluorescente, controle de intensidade luminosa.

\section{INTRODUÇÃO}

Atualmente, cerca de $20 \%$ da energia elétrica consumida mundialmente é em forma de iluminação artificial. Uma solução para redução desta taxa é a utilização de reatores eletrônicos em alta freqüência, alimentando lâmpadas fluorescentes. Além de diminuir o consumo de energia elétrica, os reatores eletrônicos apresentam vantagens comparadas aos eletromagnéticos como: maior rendimento e eficiência luminosa $(\mathrm{lm} / \mathrm{W})$, menor peso e menor volume e inexistência de "flicker" e ruído audível (Hammer, 1987; Hammer e McGowan, 1985).

A capacidade dos reatores eletrônicos na variação de intensidade luminosa "dimming" reduz o consumo de energia elétrica, o que os torna de grande importância na atual crise energética brasileira. O reator eletrônico que 
opera no modo de oscilação auto-sustentada mostrado na Figura 1, conhecido como reator eletrônico autooscilante (REAO) tem as características mencionadas acima aliadas ao baixo custo, simplicidade e confiabilidade, no entanto não se obteve até então sua aplicação em "dimming" de forma bem sucedida. O REAO tem despertado grande interesse por parte de pesquisadores com a realização de inúmeros trabalhos, devido sua aplicabilidade e pela busca de um projeto adequado para este tipo de reator (Brioschi et al., 1988; Nerone, 1995; Chang et al.,1999; Chang et al., 2001; Prado et al.,2000),

Recentemente, um reator eletrônico com circuito de comando auto-oscilante e capacidade de variação de intensidade luminosa foi proposto por (Tao et al., 2001). No entanto, o reator proposto é uma estrutura complexa que descaracteriza as principais vantagens do REAO que são: sua simplicidade, baixo custo e confiabilidade.

No presente artigo é apresentado um reator eletrônico auto-oscilante com duas propostas simples de variação de intensidade luminosa. As soluções propostas necessitam de apenas dois componentes passivos de baixa potência, agregados ao circuito de comando, mostrado na Figura 1, colocadas no braço $B_{D}$ entre os pontos 1 e 2 mostrado na Figura 2. Para facilitar a análise representa-se a rede de alimentação alternada $V_{i n}$ e a ponte retificadora $\left(D_{1}-D_{4}\right)$ juntamente com o capacitor de barramento $C_{B}$ pela fonte de tensão contínua $E$.

A solução proposta é caracterizada pela simplicidade, embora sua análise possa se tornar um tanto quanto complexa. No intuito de facilitar a análise e obter a solução desses problemas são utilizadas ferramentas de controle, como o método da função descritiva e o critério de estabilidade estendido de Nyquist. Essas ferramentas permitem o desenvolvimento de um procedimento de projeto para seleção dos parâmetros do circuito de comando auto-oscilante.

$\mathrm{O}$ artigo é organizado da seguinte forma: Na seção 2 é apresentada uma análise qualitativa do REAO bem como do REAOD. Na seção 3 desenvolve-se uma metodologia de projeto para o circuito com "dimming". Na seção 4 são apresentados os resultados experimentais. Na seção 5 são realizados alguns comentários relacionados à proposição feita e na seção 6 é apresentada a conclusão deste trabalho.

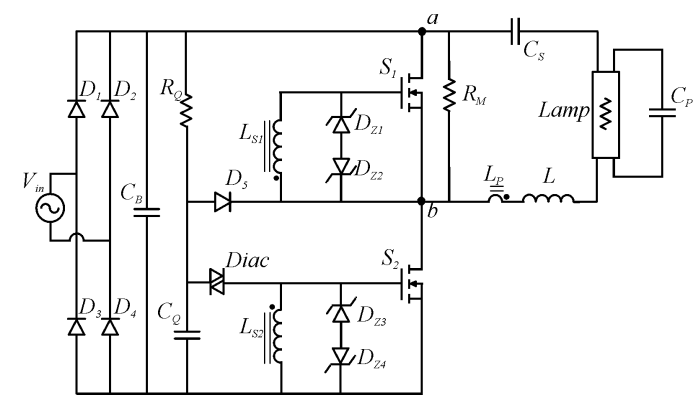

Figura 1: Reator eletrônico auto-oscilante (REAO)

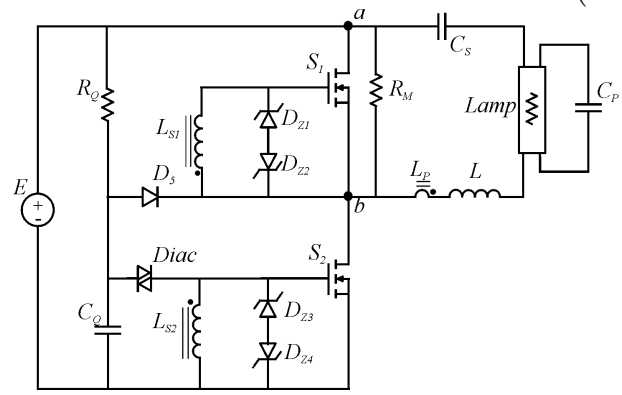

Figura 2: REAO com "dimming"(REAOD)

\section{REAO COM “DIMMING” (REAOD)}

Nesta seção, é apresentado o funcionamento do REAO tradicional. A partir deste é proposto o REAOD.

\subsection{Reator Eletrônico Auto-Oscilante}

O REAO é mostrado na Figura 1. O funcionamento desse reator baseia-se na realimentação da corrente ressonante, proveniente do filtro LCC, por meio de um transformador de corrente (TC). Os enrolamentos secundários do TC são conectados de forma complementar aos "gates" dos Mosfets $S_{1}-S_{2}$, como é mostrado na Figura 1.

A representação do circuito de "gate" pode ser feita substituindo o transformador de corrente, TC $\left(L_{P}, L_{S 1}, L_{S 2}\right)$ por uma fonte de corrente senoidal $i_{S}$ em paralelo com a sua indutância magnetizante $L_{m}$ e aproximando o diodo zener por uma fonte de tensão ideal, constante com tensão $V_{Z}$.

A partir destas aproximações pode-se considerar a corrente $i_{M}$ no indutor $L_{m}$ crescendo linearmente e a corrente do filtro LCC refletida $i_{S}$ senoidal. Portanto, a corrente zener $i_{Z}$ é constituída pela corrente magnetizante $i_{M}$ e corrente ressonante $i_{S}$ o que determina a polaridade da fonte de tensão $V_{Z}$, como é mostrado na Figura 3. 


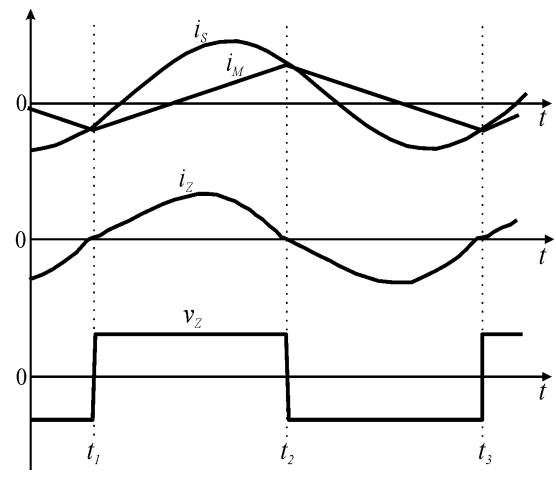

Figura 3: Formas de onda teóricas do REAO

Quando $i_{Z}$ se torna zero nos instantes $t_{1}, t_{2}$ et $t_{3}$ ocorre a troca de polaridade da tensão sobre o diodo zener, como é observado na Figura 3. A mudança da polaridade no diodo zener resulta na troca de estado dos interruptores $\left(S_{1}, S_{2}\right)$. Desse modo verifica-se que a indutância magnetizante $L_{m}$ e as tensões dos diodos zener constituemse nos elementos de maior influência na freqüência de operação do REAO.

O circuito da Figura 1 é representado por meio de diagramas de blocos, como é mostrado na Figura 4 e são feitas as seguintes simplificações: 1) a lâmpada fluorescente é modelada por uma resistência equivalente $R_{\text {Lamp }} ; 2$ ) despreza-se as capacitâncias parasitas dos Mosfets $\left(S_{1} ; S_{2}\right)$ bem como os seus tempos de abertura e fechamento; 3 ) a tensão de entrada retificada é aproximada por uma fonte de tensão contínua E; 4) a troca de estado dos interruptores pode ser representada pelo "hard limit", mostrado na Figura 4; 5) o filtro ressonante apresenta características de um filtro passa baixa atenuando as harmônicas de ordem superior.

Assim, o filtro ressonante e a lâmpada fluorescente são representados pela função de transferência $G_{F}(s)$, relacionando a corrente ressonante $I_{s}$ e tensão $V_{a b}$. A parcela do circuito relacionada com circuito de comando é representada pela função de transferência $G_{M}(s)$ que relaciona a corrente $i_{M}$ e tensão $V_{a b}$. Para análise de sistemas não lineares, como da Figura 5, pode ser utilizado o método da função descritiva e o critério estendido de Nyquist, como é mostrado a seguir.

\subsubsection{Análise do REAO por Função Descritiva}

Sistemas de controle SISO, contendo elementos não lineares que apresentam oscilações, podem ser representados por diagrama de blocos como o da Figura 5. Se as harmônicas superiores, geradas pelo elemento nãolinear "hard limit" (Figura 4), são suficientemente ate-

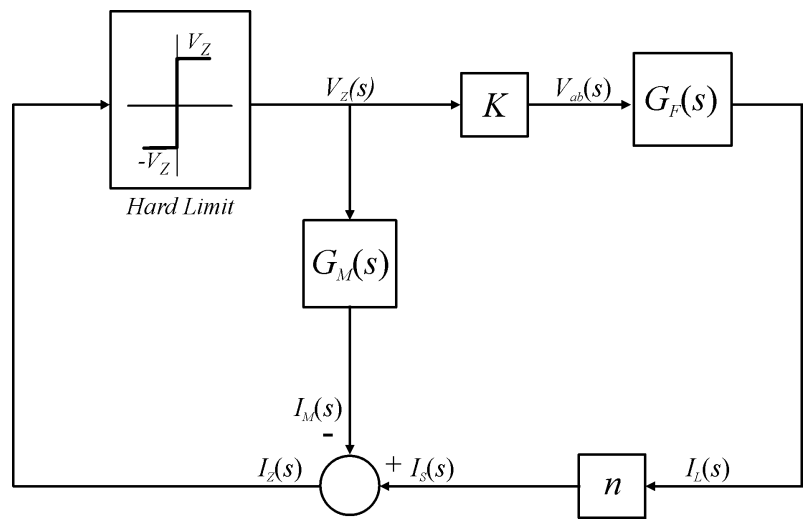

Figura 4: Representação do reator eletrônico autooscilante em forma de diagrama de blocos

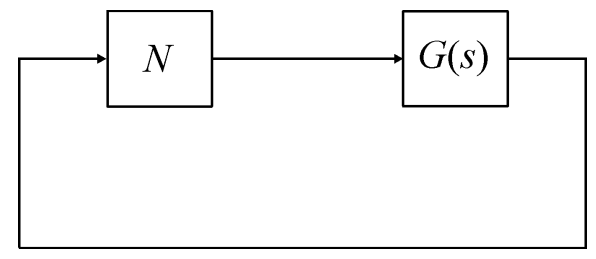

Figura 5: Diagrama de bloco do REAO reduzido

nuadas pelos elementos lineares (filtro ressonante LCC), de forma que apenas a componente harmônica fundamental de saída seja significativa, então a estabilidade do sistema pode ser prevista por uma análise de função descritiva. Assim, o "hard limit" pode ser representado pela sua função descritiva $N$ :

$$
N=\frac{2 \mathrm{E}}{\pi I_{z}}
$$

Quando existe uma oscilação auto-sustentada na saída de um sistema, a amplitude e a freqüência da oscilação podem ser determinadas a partir da representação de $G(j \omega)$. A equação característica do sistema da Figura 5 é:

$$
1+N G(j \omega)=0
$$

isolando-se $G(j \omega)$, obtém-se

$$
G(j \omega)=-\frac{1}{N} .
$$

Se (3) for satisfeita, então o sistema pode exibir um ciclo limite. Portanto, as posições relativas dos lugares geométricos $-1 / N$ e de $G(j \omega)$ dão informações de existência de auto-oscilação.

Assumindo-se que inicialmente ocorra um pequeno distúrbio no sistema com redução da amplitude $I_{Z}$ da fundamental da corrente zener $i_{Z}$, o ponto de operação se 
move de $P$ para $A$. Nota-se que o novo ponto de operação $A$ é envolvido pela curva $G(j \omega)$, no sentido indicado na Figura 6. Pelo critério estendido de estabilidade de Nyquist, a amplitude da fundamental $I_{Z}$ tende a crescer em direção a $P$. Por outro lado, se houver um distúrbio em $i_{Z}$ fazendo com que a amplitude aumente, por exemplo, para o ponto de operação $B$, este opera não envolvido por $G(j \omega)$, resultando um comportamento estável, de maneira que a amplitude se reduz tendendo novamente ao ponto $P$. Portanto, de acordo com o critério estendido de Nyquist, o ponto $P$ representa uma possível oscilação auto-sustentada conforme (Pinheiro et al., 1999). Ainda, através da solução das equações do circuito para o ponto $P$ é possível determinar os parâmetros adequados do funcionamento do REAO.

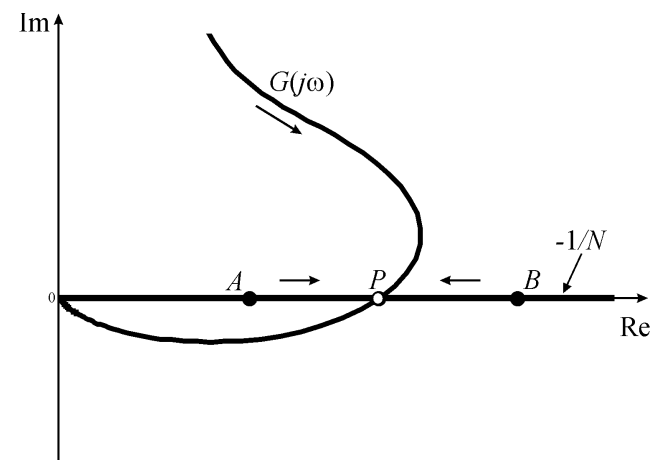

Figura 6: Diagrama de Nyquist para o REAO

\subsection{Reator Eletrônico Auto-Oscilante com “Dimming" (REAOD)}

Na seção anterior foi demonstrado que $i_{Z}$ determina a frequiência de operação do REAO, sendo $i_{Z}$ dependente de $V_{Z}$ e $L_{m}$. Para variar a frequiência, deve-se alterar $i_{Z}$, no entanto $V_{Z}$ e $L_{m}$ são elementos que não permitem a sua alteração durante o funcionamento do REAO, restando então a alternativa de variar $i_{Z}$ por meio de um circuito adicional, mostrado na Figura 7. Desta forma, a variação da freqüência de operação fica restrita a mudança da fase de $i_{Z}$, possibilitada pelo braço $\mathrm{B}_{D}$ agregado ao circuito de comando mostrado na Figura 2. Duas configurações típicas para $\mathrm{B}_{D}$ são mostrados na Figura 7(a) e Figura 7(b), discutidas a seguir.

\subsubsection{Análise Qualitativa do REAOD com a rede LR:}

A utilização de um circuito LR série permite aumentar a inclinação da corrente resultante $I_{D}$ (Figura 8), dada pela soma de $I_{M}$ e a corrente de $B_{D}\left(I_{B D}\right)$, como mostra a 3.1. Esta mudança altera o período em que ocorre a troca de estado, o que resulta no aumento da freqüência

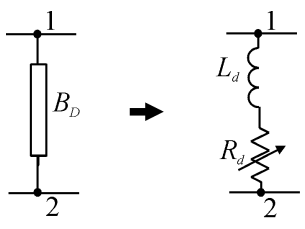

(a)

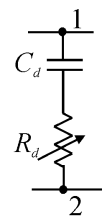

(b)
Figura 7: Braço $B_{D}$ do REAOD com rede (a)LR e (b)CR

de auto-oscilação mostrada pelo ponto $A_{L}$, na 3.1. Portanto, quanto menor o resistor $R_{d}$, maior é a influência de $I_{B}$, conseqüentemente a freqüência de operação do conversor aumenta reduzindo a potência da lâmpada.

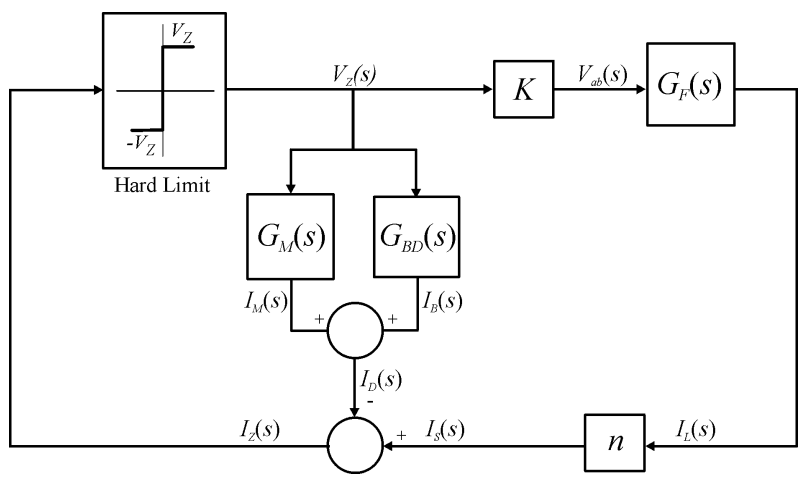

Figura 8: Diagrama de blocos do REAOD

\subsubsection{Análise Qualitativa do REAOD com rede CR:}

Ao contrário do primeiro, a utilização da rede CR permite diminuir a inclinação da corrente resultante $I_{D}$, como mostra a 3.1. Esta mudança altera o período da troca de estado, o que resulta na redução da freqüência de auto-oscilação mostrada pelo ponto $A_{C}$, na 3.1 . Portanto, quanto menor for o resistor $R_{d}$, menor é $I_{B}$, conseqüentemente a freqüência de operação do conversor diminui, aumentando a potência da lâmpada.

As análises feitas nessas subseções permitem o entendimento do funcionamento do REAOD. No entanto, para o projeto do REAOD é necessário analisá-lo como um sistema de controle SISO, o que permite obter equações de projeto para os componentes do circuito de comando, mostrado na próxima seção.

\section{PROCEDIMENTO DE PROJETO}

Nesta etapa será representado o REAOD como um sistema de controle SISO não linear do tipo relé. Um procedimento de projeto será desenvolvido em etapas para 
os dois circuitos utilizados: REAOD com rede LR e REAOD com rede CR.

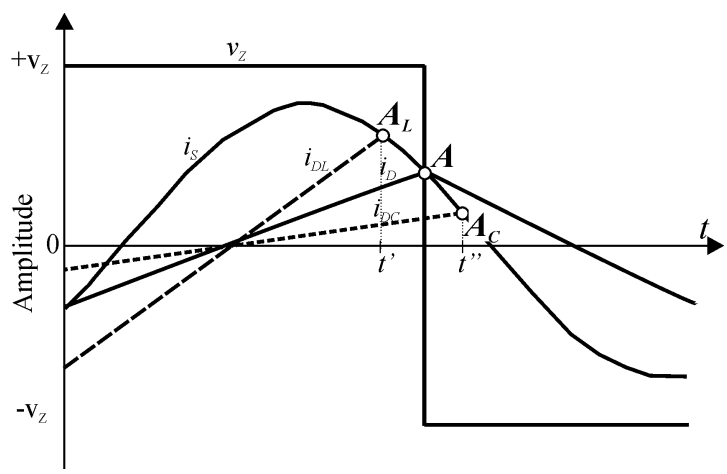

Figura 9: Formas de onda teóricas para o REAO com as redes $\mathrm{LR}$ e CR

\subsection{REAOD com Rede LR}

\subsubsection{Projeto do Filtro Ressonante:}

Primeiramente, especifica-se a freqüência de operação para a potência nominal. O projeto do filtro ressonante LCC é baseado na aproximação fundamental e no ângulo de defasagem da corrente do filtro, em relação à tensão aplicada ao mesmo. Este procedimento não será mostrado devido o mesmo já ser conhecido em (Prado et al., 2001).

\subsubsection{Escolha da Faixa de Freqüência de operação do REAOD:}

Nesta etapa define-se a faixa de freqüência de operação do REAOD que garante a operação ZVS do conversor. Através da Figura 10, visualiza-se a variação da potência pela freqüência, identificando-se as freqüências de operação máximas, $\omega_{\max }$ e mínimas, $\omega_{\min }$, que estão associadas aos níveis de potências de operação da lâmpada fluorescente.

Para projeto da rede LR as condições da Tabela 1 devem ser satisfeitas. Portanto, escolhendo-se a freqüência de chaveamento mínima $\omega=\omega_{\text {min }}$ maior que a freqüência de ressonância do filtro ressonante LCC, garante-se comutação ZVS para toda faixa de freqüência de operação.

\subsubsection{Determinação da Indutância Magnetizante $\mathbf{L}_{m}$ :}

O valor da indutância $L_{m}$ esta relacionado com a freqüência de operação do REAOD, sem a influência de
Tabela 1: Condições de Projeto para Rede LR

\begin{tabular}{|c|c|}
\hline \multicolumn{2}{|c|}{ Mínima influência do braço $B_{D}$} \\
\hline$\omega$ & $\omega_{m n}$ \\
\hline$R_{d}$ & $\infty$ \\
\hline$P$ & $P_{\max }=$ Potência máxima na lâmpada \\
\hline \multicolumn{2}{|c|}{ Máxima influência do braço $B_{D}$} \\
\hline$\omega$ & $\omega_{\max }$ \\
\hline$R_{d}$ & 0 \\
\hline$P$ & $P_{\min }=$ Potência mínima na lâmpada \\
\hline
\end{tabular}

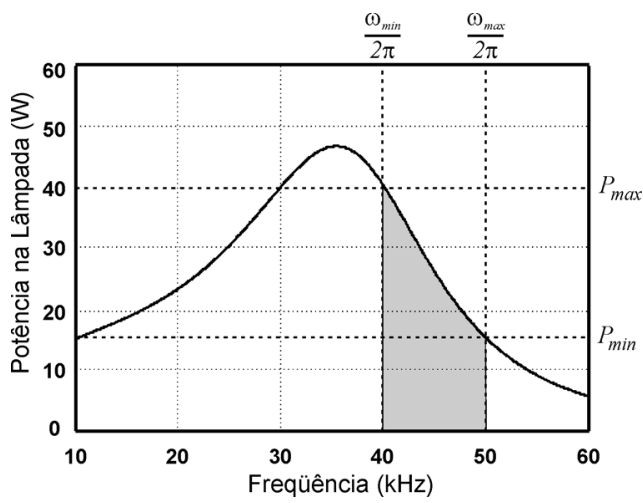

Figura 10: Resposta em freqüência do filtro LCC ressonante versus potência na lâmpada fluorescente

$\mathrm{B}_{D}\left(R_{d}=\infty\right)$. Para sua determinação definem-se as seguintes expressões:

$$
\begin{gathered}
G_{F}(s)=\frac{1}{L} \frac{s^{2}+a s}{s^{3}+a s^{2}+b s+c}, \\
G_{M}(s)=\frac{1}{L_{m} s} \\
G_{B D}(s)=G_{L R}(s)=\frac{1}{L_{d} s+R_{d}},
\end{gathered}
$$

$$
G(s)=K G_{F}(s) n-\left(G_{M}(s)+G_{D B}(s)\right),
$$

onde: $G_{F}(s)=V_{A B}(s) / I(s)$ por $s=j \omega, a=1 /\left(R C_{p}\right)$, $b=1 /\left(L\left(C_{s}+C_{p}\right)\right), c=1 /\left(R C_{p} C_{s} L\right), K=E /\left(2 V_{Z}\right) \mathrm{e}$ $R=R_{\text {Lmap }}$.

O diagrama de bloco da Figura 4 pode ser representado na forma da Figura 5 e reduzindo as expressões deriva- 
das do mesmo, na seguinte forma temos:

$$
\operatorname{Im}\left(K G_{F}(j \omega) n\right)=\frac{K n}{L} \frac{\left[a \omega^{2}\left(a \omega^{2}-c\right)+\omega^{3}\left(b \omega-\omega^{3}\right)\right]}{\left(\left(c-a \omega^{2}\right)^{2}+\left(b \omega-\omega^{3}\right)^{2}\right)},
$$

$$
\begin{gathered}
K_{F}(\omega)=\frac{K n}{L} \frac{\left[a \omega^{2}\left(a \omega^{2}-c\right)+\omega^{3}\left(b \omega-\omega^{3}\right)\right]}{\left(\left(c-a \omega^{2}\right)^{2}+\left(b \omega-\omega^{3}\right)^{2}\right)}, \\
K_{M}(\omega)=\operatorname{Im}\left(G_{M}(j \omega)\right)=-\frac{1}{L_{m} \omega}, \\
K D_{L R}(\omega)=\operatorname{Im}\left(G_{B D}(j \omega)\right)=\frac{-L_{d} \omega}{R_{d}^{2}+L_{d}^{2} \omega^{2}}
\end{gathered}
$$

O cálculo de $L_{m}$ é feito considerando a mínima influência do braço $\mathrm{B}_{D}$ (Tabela 1). A solução de $L_{m}$ passa a ser um problema algébrico, sendo esta determinada pela igualdade da expressão $\operatorname{Im}(G(j \omega))=\operatorname{Im}\left(G_{F}(j \omega)\right.$ $\left(G_{M}(j \omega)+\left(G_{D B}(j \omega)\right)=0\right.$, considerando $G_{D B}(j \omega)=0$, resulta em:

$$
\lim _{R_{d} \rightarrow \infty}\left(K_{F}(\omega)-K_{M}(\omega)-K D_{L R}(\omega)\right)=0,
$$

como resultado, $L_{m}$ é definido por:

$$
L_{m}=\frac{-1}{\omega K_{F}(\omega)}
$$

em que: $n=n_{P} / n_{S}$, e $\mathrm{n}_{S}$ é o número de espiras do enrolamento primário e $n_{P}$ é o número de espiras do enrolamento secundário do TC.

\subsubsection{Projeto da Indutância $\mathrm{L}_{d}$ para o REAOD:}

Para solução dos elementos que constituem o braço $B_{D}$ do REAOD considera-se a condição de máxima influência de $B_{D}\left(R_{d}=0\right)$ determinada na seção 3.1.1.2 para $\omega=\omega_{\max }$. Reduzindo o diagrama de blocos da Figura 8, na forma do diagrama da Figura 5 , determina-se $L_{d}$ matematicamente igualando-se $\operatorname{Im}(G(j \omega))=\operatorname{Im}\left(G_{F}(\mathrm{j} \omega)\right.$ $\left.\left(\mathrm{G}_{D}(j \omega)+G_{B D}(\mathrm{j} \omega)\right)\right)=0$ e de acordo com

$$
\lim _{R_{d} \rightarrow 0}\left(K_{F}(\omega)-K_{M}(\omega)-K D_{L R}(\omega)\right)=0,
$$

onde:

$$
L_{d}=\frac{1}{\omega\left(K_{M}(\omega)-K_{F}(\omega)\right)} .
$$

\subsection{REAOD com Rede CR}

\subsubsection{Projeto do Filtro Ressonante:}

A escolha da freqüência e o projeto do filtro ressonante LCC são análogos ao procedimento adotado para a rede LR em 3.1.1.1

\subsubsection{Escolha da Faixa de Freqüência de Operação do REAOD}

Analogamente em 3.1.1.2. deve-se garantir a operação em ZVS para toda a faixa de freqüência de operação do REAOD. Para projeto as condições da Tabela 2 devem ser satisfeitas. Portanto, escolhendo a freqüência de chaveamento mínima $\omega=\omega_{\text {min }}$ maior que a freqüência de ressonância do filtro ressonante LCC,g garante-se comutação ZVS para toda faixa de freqüência de operação.

Tabela 2: Condições de Projeto para Rede CR

\begin{tabular}{|c|c|}
\hline \multicolumn{2}{|c|}{ Mínima influência do braço $\boldsymbol{B}_{D}$} \\
\hline$\omega$ & $\omega_{\max }$ \\
\hline$R_{d}$ & $\infty$ \\
\hline$P$ & $P_{\min }=$ Potência mínima na lâmpada \\
\hline \multicolumn{2}{|c|}{ Máxima influência do braço $\boldsymbol{B}_{D}$} \\
\hline$\omega$ & $\omega_{\min }$ \\
\hline$R_{d}$ & 0 \\
\hline$P$ & $P_{\max }=$ Potência nominal na lâmpada \\
\hline
\end{tabular}

\subsubsection{Determinação da Indutância Magnetizante $\mathbf{L}_{m}$ :}

Como a definição de $L_{m}$ relaciona-se com a freqüência de operação do REAOD sem a influência de $B_{D}$ a expressão de $L_{m}$ é a mesma que em 3.1.1.3, definida por 3.1.4 considerando $\left(R_{d}=\infty\right)$, sendo $G_{B D}(\mathrm{j} \omega)$ definido por:

$$
G_{B D}(s)=G_{C R}(s)=\frac{C_{d} s}{R_{d} C_{d} s+1},
$$

isolando a parte imaginária de 3.2.4 tem-se:

$$
\begin{aligned}
K D_{C R}(\omega) & =\operatorname{Im}\left(G_{B D}(j \omega)\right)=\operatorname{Im}\left(G_{C R}(j \omega)\right) \\
& =\frac{C_{d} \omega}{1+\left(R_{d} C_{d} \omega_{d}\right)^{2}} .
\end{aligned}
$$

\subsubsection{Projeto da Capacitância $\mathrm{C}_{d}$ para o REAOD:}

A solução de $C_{d}$ é feita de forma análoga a de $L_{d}$, no entanto a condição definida para o projeto de $C_{d}$ que garante operação em ZVS é $\omega=\omega_{m i n}$, o que resulta em:

$$
\lim _{R_{d} \rightarrow 0}\left(K_{F}(\omega)-K_{M}(\omega)-K D_{C R}(\omega)\right)=0,
$$

como resultado, $C_{d}$ é encontrado por

$$
C_{d}=\frac{K_{F}(\omega)-K_{M}(\omega)}{\omega} .
$$


No entanto, as soluções dos parâmetros apresentados nos passos anteriores não garantem o funcionamento em modo de auto-oscilação do REAOD, sendo necessário verificar a estabilidade relacionada aos elementos determinados no projeto. O item a seguir relaciona os elementos determinados a estabilidade do reator.

\subsection{Estabilidade Relacionada com a Opera- ção do REAOD}

Para validar o cálculo de $L_{m}$ traça-se o diagrama de Nyquist, como mostrado na Figura 6. Verifica-se que o ponto $P$ associado à freqüência de auto-oscilação é um ponto possível de oscilação auto-sustentada, uma vez que satisfaz as condições previamente estabelecidas em 2.1.1. Além disso, é necessário verificar a estabilidade na inclusão de $L_{d}$ no circuito.

Para isto, verificam-se os possíveis pontos de operação, à medida que a freqüência se modifica. Então, plotam-se curvas com valores de resistência dimming $R_{d}$ mínima e máxima, com diferentes resistências $R_{\text {Lamp }}$ para avaliar a estabilidade do REAOD em várias condições de operação. As Figura 11 e Figura 12 representam essas variações no REAOD com a rede $L R$, sendo a primeira para uma condição de resistência "dimming" $R_{d}$ mínima e a segunda para $R_{d}$ máxima.

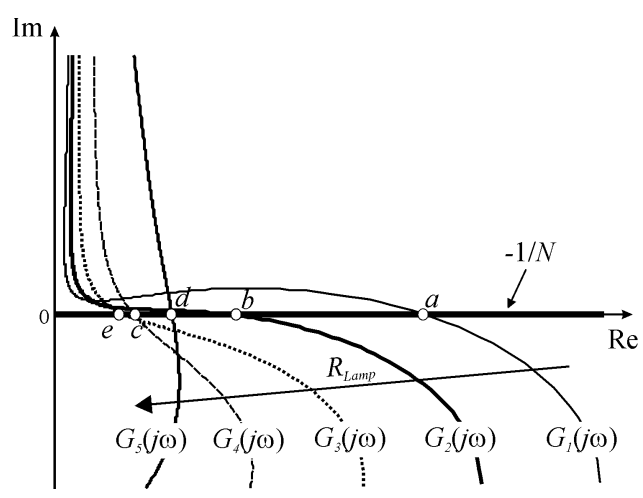

Figura 11: Diagrama de Nyquist para o REAO com a rede $\mathrm{LR}$ nas condições de $\mathrm{R}_{d}$ mínimo

As interseções " $a$ "- $d$ " das curvas $-1 / \mathrm{N}$ e $G_{1}(j \omega)-G_{5}(j \omega)$ fornecem informações sobre prováveis pontos de oscilação auto-sustentada, pois atendem aos critérios definidos em 2.1.1. Da mesma forma, a rede CR deve apresentar as características apresentadas na análise da rede LR, para que exista oscilação auto-sustentada em todos os casos. Esta análise é suprimida, em virtude de ser similar a análise apresentada para rede LR.

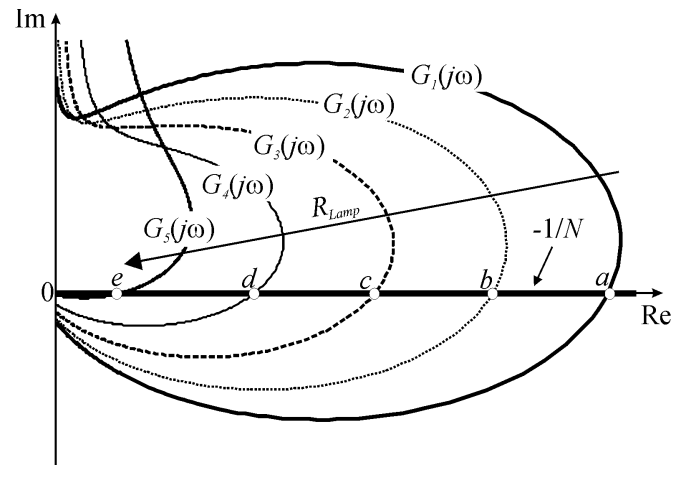

Figura 12: Diagrama de Nyquist para o REAO com a rede $\mathrm{LR}$ nas condições de $\mathrm{R}_{d}$ máximo

\section{RESULTADOS EXPERIMENTAIS}

Tabela 3: Resumo dos Parâmetros Parâmetros do Filtro Ressonante

\begin{tabular}{|c|c|}
\hline \multicolumn{2}{|c|}{ Parâmetros do Filtro Ressonante } \\
\hline$C_{S}$ & $\begin{array}{c}\text { Capacitor de Polipropileno, } \\
147 \mathrm{nF} / 250 \mathrm{Vac}\end{array}$ \\
\hline$C_{P}$ & $\begin{array}{c}\text { Capacitor de polipropileno, } \\
10 \mathrm{nF} / 600 \mathrm{Vac}\end{array}$ \\
\hline$L$ & $\begin{array}{c}\text { Indutor, } 800 \mu \mathrm{H}, 150 \text { espiras, } \\
\text { núcleo EE20 IP6-Thornton }\end{array}$ \\
\hline \multicolumn{2}{|c|}{ Parâmetros do Circuito de Comando } \\
\hline$\overline{L_{m}}($ rede LR $)$ & $\begin{array}{c}\mathrm{L}_{m}=688 \mu \mathrm{H} .: \mathrm{n}: 2 / 12 / 12 \text { espiras, } \\
\text { núcleo T15 IP6-Thornton }\end{array}$ \\
\hline$L_{m}($ rede $\mathrm{CR})$ & $\begin{array}{c}\mathrm{L}_{m}=570 \mu \mathrm{H} .: n: 2 / 6 / 6 \text { espiras, } \\
\text { núcleo T15 IP6-Thornton }\end{array}$ \\
\hline $\mathrm{D}_{Z 1-4}$ & |p184pt|Diodos zener $12 \mathrm{~V} 1 / 2 \mathrm{~W}$ \\
\hline Diac & DB3 \\
\hline $\mathrm{R}_{Q}$ & Resistor $220 \mathrm{k} \Omega / 1 / 8 \mathrm{~W}$ \\
\hline $\mathrm{C}_{Q}$ & Capacitor cerâmico $100 \mathrm{nF} / 63 \mathrm{~V}$ \\
\hline $\mathrm{R}_{M}$ & Resistor $470 \mathrm{k} \Omega / 1 / 8 \mathrm{~W}$ \\
\hline \multicolumn{2}{|r|}{ Outros } \\
\hline $\mathrm{S}_{1}, \mathrm{~S}_{2}$ & MOSFET's IRF740 \\
\hline $\mathrm{D}_{1}-\mathrm{D}_{5}$ & Diodo 1N4004 \\
\hline$\overline{\mathrm{R}_{\text {Lamp }}}$ & Lâmpada Fluorescente Tubular 40W \\
\hline $\mathrm{C}_{B}$ & $\begin{array}{c}\text { Capacitor Eletrolítico } \\
100 \mu \mathrm{F} / 200 \mathrm{Vdc}\end{array}$ \\
\hline \multicolumn{2}{|c|}{ Componentes do Dimming } \\
\hline$L_{d}$ & Indutor dimming, $150 \mathrm{uH}$ \\
\hline$C_{d}$ & Capacitor dimming, $47 \mathrm{nF} / 30 \mathrm{~V}$ \\
\hline$R_{d}$ & Resistor variável, $1 \mathrm{k} \Omega$ \\
\hline
\end{tabular}

A partir do procedimento de projeto desenvolvido construiu-se os protótipos, cujos parâmetros estão resumidos nas Tabelas 3 e 4 . Os resultados experimentais de um REAOD alimentando uma lâmpada fluorescente de $40 \mathrm{~W}$ são apresentados para as duas variações pro- 
Tabela 4: Dados de Entrada

\begin{tabular}{|c|c|}
\hline $\begin{array}{c}\text { Tensão de entrada } \\
\text { Potência de Saída }\end{array}$ & $\begin{array}{c}\mathrm{V}_{i n}=110 \mathrm{Vrms}, 60 \mathrm{~Hz} \\
\mathrm{P}=40 \mathrm{~W}\end{array}$ \\
\hline Freqüência de chaveamento & $\mathrm{F}=40 \mathrm{kHz}$ \\
\hline Tensão do diodo zener & $\mathrm{V}_{Z}: 12 \mathrm{~V}$ \\
\hline Resistência da Lâmpada & $\mathrm{R}=270 \Omega$ \\
\hline
\end{tabular}

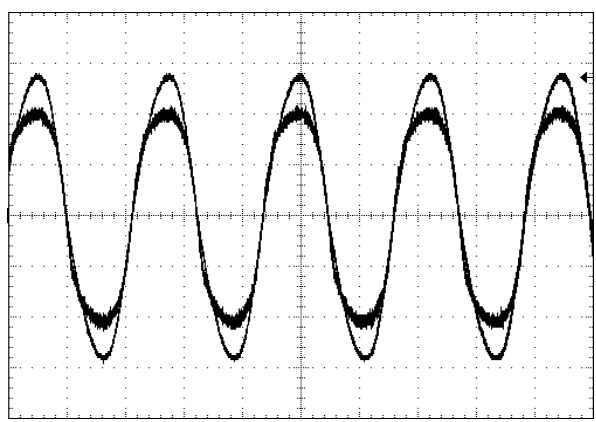

(a) $50 \mathrm{~V} / \operatorname{div}, 200 \mathrm{~mA} / \operatorname{div} ; 12,5 \mu \mathrm{s} /$ div; potência máxima $32 \mathrm{~W}$

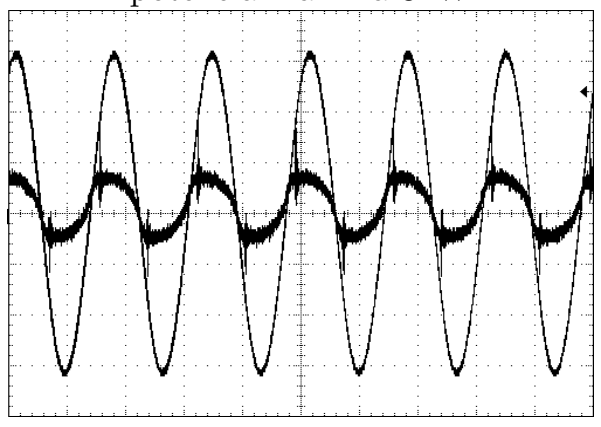

(b) $50 \mathrm{~V} / \operatorname{div}, 200 \mathrm{~mA} / \operatorname{div} ; 12,5 \mu \mathrm{s} / \operatorname{div}$; potência mínima de $10 \mathrm{~W}$

Figura 13: Resultados experimentais do REAOD com a rede LR: (a) e (b) Tensão e corrente na lâmpada

postas de rede $\mathrm{LR}$ e CR.

Resultados experimentais para o REAOD com a rede LR são apresentados na Figuras 13 e 14. Nas Figuras 13 (a) e (b) são mostradas as formas de onda da tensão e corrente na lâmpada fluorescente nas condições de potência máxima e mínima, respectivamente. Nas Figuras 14 (a) e (b) são mostradas as formas de onda da tensão e corrente em um dos interruptores a nas condições de potência máxima e mínima, respectivamente. Estas formas de onda demonstram a comutação ZVS em todas condições de operação.

\section{DISCUSSÕES FINAIS}

Resultados experimentais para o REAOD com a rede CR são apresentados na Figuras 15 e 16. Nas Figuras

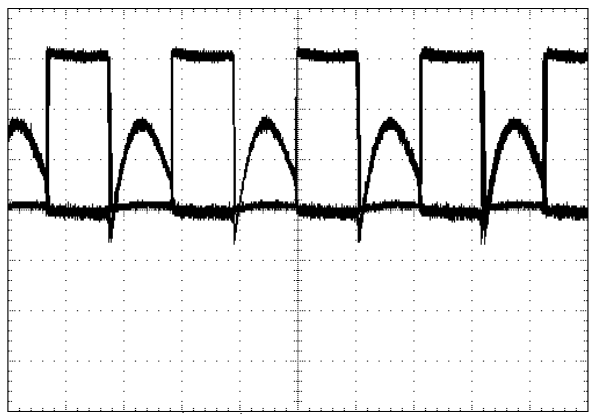

(a) $50 \mathrm{~V} / \operatorname{div}, 500 \mathrm{~mA} / \operatorname{div} ; 12.5 \mu \mathrm{s} / \operatorname{div}$, potência máxima $32 \mathrm{~W}$

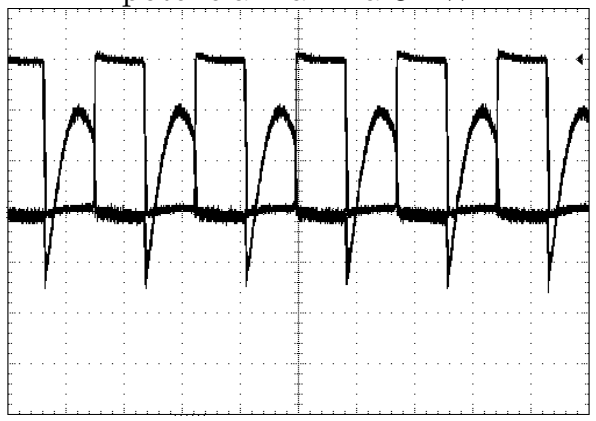

(b) $50 \mathrm{~V} /$ div; $500 \mathrm{~mA} /$ div; $12.5 \mu \mathrm{s} /$ div; potência mínima $10 \mathrm{~W}$

Figura 14: Resultados experimentais do REAOD com a rede LR: (a) e (b) Tensão e corrente em um dos interruptores

15(a) e (b) são mostradas as formas de onda da tensão e corrente na lâmpada para potência máxima e mínima, respectivamente.

Nas Figuras 16 (a) e (b) são mostradas as formas de onda da tensão e corrente em um dos interruptores nas condições de potência máxima e mínima, mostrando também a operação em ZVS do conversor para todas as condições de carga.

O controle de intensidade luminosa de lâmpadas fluorescentes para o REAOD com a rede LR e CR é resumido pelos gráficos de potência versus freqüência, que são mostrados nas Figuras 17(a) e 18(a) respectivamente. Pode-se observar através dos resultados apresentados a faixa de variação de potência na lâmpada que se reflete na variação da intensidade luminosa da mesma. Nas Figuras 17 (b) e 18(b) são mostradas a variação do fator de crista de corrente na lâmpada versus freqüência para o REAOD para as redes LR e CR respectivamente.

Os resultados apresentados demonstram a viabilidade do reator eletrônico auto-oscilante proposto no controle de intensidade luminosa de lâmpadas fluorescentes. A 


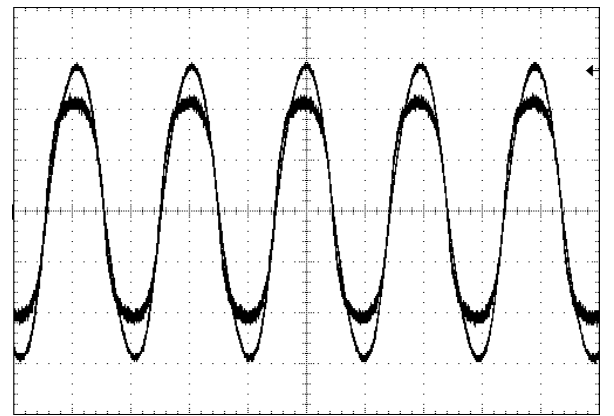

(a) $50 \mathrm{~V} / \operatorname{div}, 200 \mathrm{~mA} / \operatorname{div} ; 12.5 \mu \mathrm{s} /$ div; potência máxima $41 \mathrm{~W}$

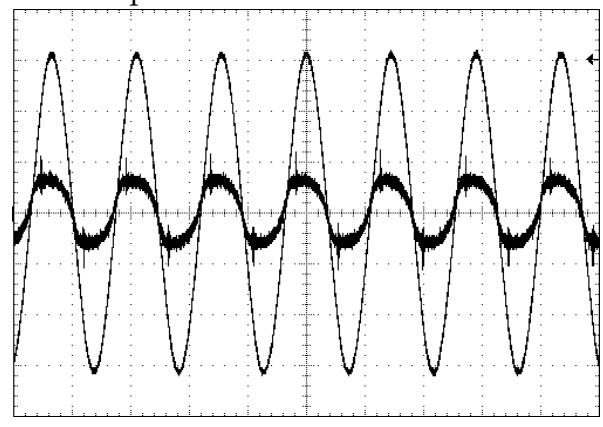

(b) $50 \mathrm{~V} /$ div, $200 \mathrm{~mA} /$ div; $12.5 \mu \mathrm{s} /$ div; potência mínima $16 \mathrm{~W}$

Figura 15: Resultados experimentais do REAOD com a rede CR: (a) e (b)Tensão e corrente na lâmpada

metodologia de projeto empregada e a forma de variação da freqüência podem ser estendidas a outras aplicações que utilizam o circuito de comando auto-oscilante.

As principais características do REAOD proposto são relacionadas a seguir, destacando-se:

- Simplicidade: relacionada ao indutor da rede LR (ou capacitor com a rede $\mathrm{CR}$ ) utilizado no circuito de comando de forma independente, isto é, sem acoplamento entre $L_{d}$ (ou $C_{d}$ ) com o transformador de corrente TC, ao contrário da proposição proposta por (Tao et al., 2001).

- Comutação ZVS: garantida analisando as características de cada rede ( $\mathrm{LR}$ ou $\mathrm{CR}$ ), relacionando as faixas de freqüências que devem ser atendidas para manter a comutação ZVS mostradas nas Tabelas 1 e 2. Sendo a freqüência de ressonância do filtro conhecida em função do projeto do mesmo segundo (Prado et al. 2000 e Prado et al. 2001), torna-se possível então, através de uma metodologia de projeto adequada, manter a freqüência de chaveamento do reator eletrônico sempre em um valor superior à

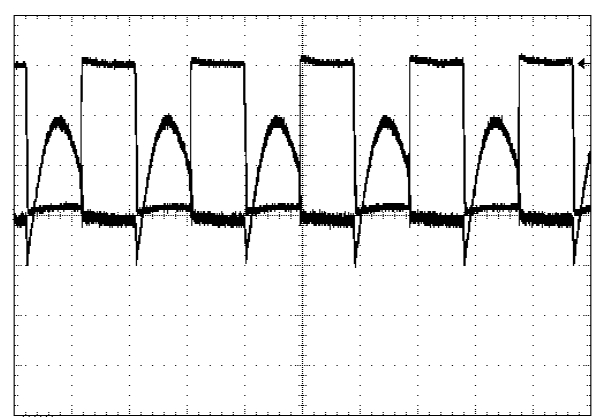

(a) $50 \mathrm{~V} /$ div; $500 \mathrm{~mA} /$ div; $10 \mu \mathrm{s} /$ div; potência máxima $41 \mathrm{~W}$

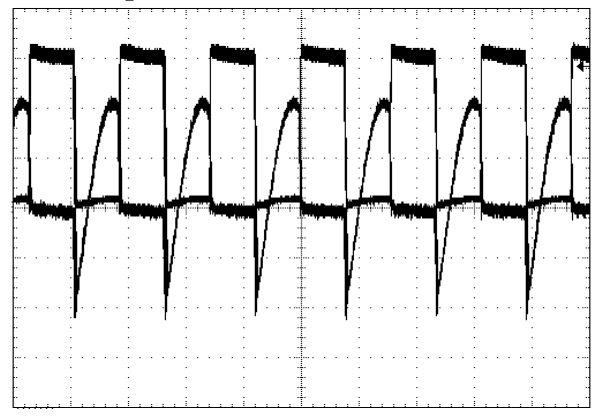

(b) $50 \mathrm{~V} /$ div; $500 \mathrm{~mA} /$ div; $12,5 \mu \mathrm{s} /$ div; potência mínima $16 \mathrm{~W}$

Figura 16: Resultados experimentais do REAOD com a rede CR: (a) e (b)Tensão e corrente em um dos interruptores

freqüência de ressonância, garantindo a comutação ZVS

- Praticabilidade: Além de variar a intensidade luminosa do REAO, a confiabilidade e o baixo custo do REAO são garantidas pela utilização de elementos passivos de baixa potências, em ambas as variações (REAOD com rede LR ou REAOD com rede CR).

\section{CONCLUSÃO}

Um reator eletrônico com duas propostas simples para variação de intensidade luminosa foi concebido, através de sua análise, empregando ferramentas de controle. Uma simples alteração no seu circuito de comando permitiu controlar a intensidade luminosa de uma lâmpada fluorescente, variando a freqüência de chaveamento do reator eletrônico auto-oscilante. A análise do REAOD como um sistema de controle SISO, a utilização da função descritiva e o critério estendido de Nyquist deram suporte na elaboração e no sucesso no projeto do REAOD. Isto é evidenciado pelos resultados experimentais que mostraram a variação da potência da lâmpada e 


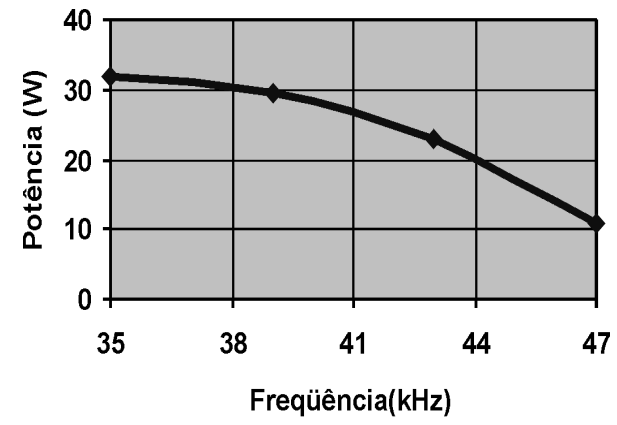

(a)

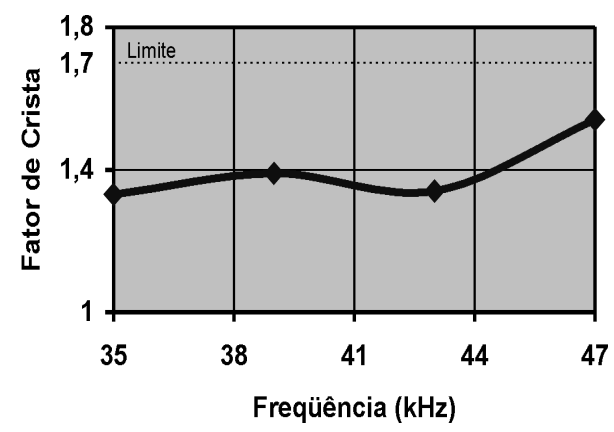

(b)

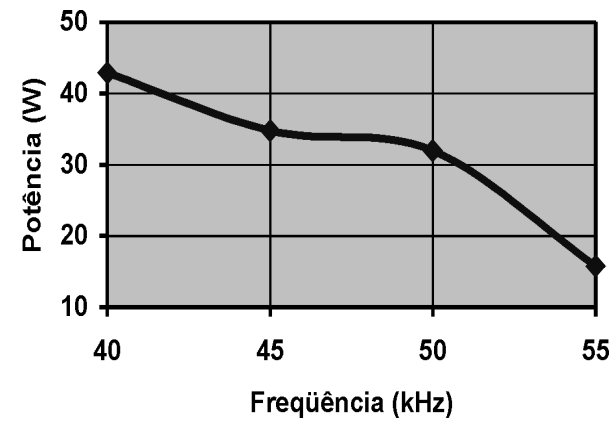

(a)

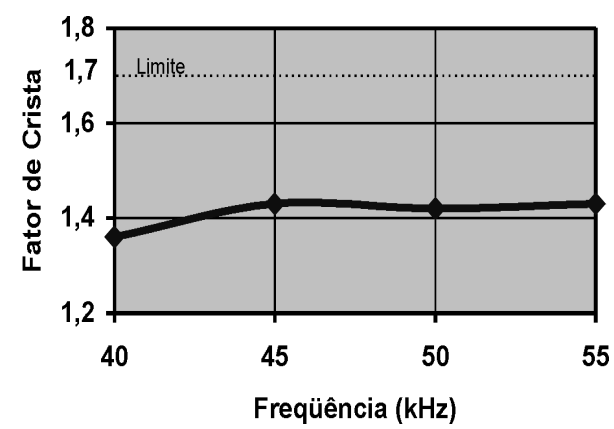

(b)
Figura 17: Gráficos do REAOD com a rede LR para variação da: (a) potência da lâmpada versus freqüência e (b) fator de crista da corrente da lâmpada versus freqüência

comutação em ZVS para toda a faixa de potência de operação do reator. Portanto, obteve-se, de forma inédita, um reator eletrônico auto-oscilante com a possibilidade de variação de intensidade luminosa de forma viável, sem comprometer as principais características do REAO que são o baixo custo, sua simplicidade e grande confiabilidade.

\section{AGRADECIMENTOS}

Os autores agradecem à THORNTON INPEC, pelo apoio com núcleos utilizado nas montagens, à CAPES e CNPq pelo apoio financeiro.

\section{REFERÊNCIAS}

Brioschi, R. O., Lamego M. M. e Vieira, J. L. F. (1998). "Reator eletrônico de Baixo Custo e Alto Fator de Potência," SBA Controle $\&$ Automação, Vol. 9, No 3, pp. 149-155.

Chang, C., Chang, J., and Bruning, G. W. (1999). "Analysis of the Self-Oscillating Series Resonant In-
Figura 18: Gráficos do REAOD com a rede CR para variações de: (a) potência da lâmpada fluorescente versus freqüência e (b) fator de crista da corrente da lâmpada fluorescente versus freqüência

verter for Electronic Ballasts," IEEE Transactions on Power Electronics, Vol. 14, No. 3, pp. 533-540.

Chang, C. and Bruning, G. W. (2001). "Self-Oscillating Electronic Ballast Analysis Via Relay Systems Approach," IEEE Transactions on Industry Applications, Vol. 37, No. 1, pp 255-261.

Hammer, E. E.(1987). "High Frequency Characteristics of Fluorescent Lamps up to $500 \mathrm{kHz}$," Journal of the Illuminating Engineering Society, pp.52-61.

Hammer, E. E. e McGowan, T. K. (1985). "Characteristics of Various F40 Fluorescent Systems at $60 \mathrm{~Hz}$ and High Frequency," IEEE Transactions on Industry Applications, Vol. 21, No. 1, pp. 11-16.

Nerone, L. R. (1995). "A Mathematical Model of the Class D Converter for Compact Fluorescent Ballasts," IEEE Transaction on Power Electronics, Vol. 10, No. 6. pp. 708-715.

Pinheiro, H., Jain, P. K. e Joós, G. (1999) "Selfsustained Oscillating Resonant Converters Opera- 
ting Above the resonant Frequency," IEEE Transaction on Power Electronics, Vol. 14 pp.803-815.

Prado, R. N., Seidel, A. R., Bisogno, F. E. e Costa, M. A. D. (2000). "A Design Method for Electronic Ballast for Fluorescent Lamps," IEEE IECON proc, record.

Prado, R. N., Seidel, A. R., Bisogno, F. E., Pavão e R. K. (2001) "Self-oscillating Electronic Ballast Design based on Point of View of Control System," IEEE IAS Proc, record.

Tao, F., Zhao, Q., Lee, F. C. e Naoki, O. (2001) "SelfOscillating Electronic Ballast with Dimming Control," IEEE PESC Proc., record. 